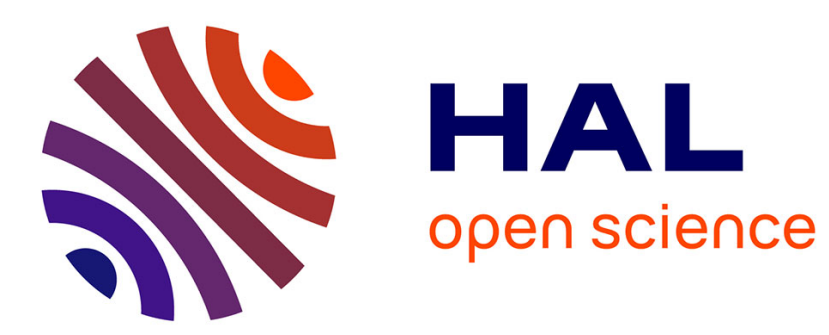

\title{
Real time ultrasound image denoising
}

Fernanda Palhano Xavier de Fontes, Guillermo Andrade Barroso, Pierrick

Coupé, Pierre Hellier

\section{To cite this version:}

Fernanda Palhano Xavier de Fontes, Guillermo Andrade Barroso, Pierrick Coupé, Pierre Hellier. Real time ultrasound image denoising. Journal of Real-Time Image Processing, 2010, 10.1007/s11554-0100158-5 . inria-00476122v2

\section{HAL Id: inria-00476122 https://hal.inria.fr/inria-00476122v2}

Submitted on 21 May 2010

HAL is a multi-disciplinary open access archive for the deposit and dissemination of scientific research documents, whether they are published or not. The documents may come from teaching and research institutions in France or abroad, or from public or private research centers.
L'archive ouverte pluridisciplinaire HAL, est destinée au dépôt et à la diffusion de documents scientifiques de niveau recherche, publiés ou non, émanant des établissements d'enseignement et de recherche français ou étrangers, des laboratoires publics ou privés. 
Fernanda Palhano Xavier de Fontes .

Guillermo Andrade Barroso .

Pierre Hellier

\title{
Real time ultrasound image denoising
}

the date of receipt and acceptance should be inserted later

\begin{abstract}
Image denoising is the process of removing the noise that perturbs image analysis methods. In some applications like segmentation or registration, denoising is intended to smooth homogeneous areas while preserving the contours. In many applications like video analysis, visual servoing or image-guided surgical interventions, real-time denoising is required. This paper presents a method for real-time denoising of ultrasound images: a modified version of the NL-means method is presented that incorporates an ultrasound dedicated noise model, as well as a GPU implementation of the algorithm. Results demonstrate that the proposed method is very efficient in terms of denoising quality and is real-time.
\end{abstract}

\section{Introduction}

Image denoising is a key component of image processing workflows. Denoising aims at reducing the noise in homogeneous areas while preserving the image contours. Denoising is important for postprocessing methods like segmentation, classification, object recognition, pattern analysis, registration, etc. In this context the denoising of ultrasound images is particularly challenging due to the particular texture of the ultrasound images. The noise, often referred to as "speckle", is a multiplicative signal-dependent noise. Ultrasound is a medical imaging modality suited for many applications, since the image acquisition is real-time. Therefore, ultrasound can be used for surgical guidance and robotic assisted interventions. For surgical applications, ultrasound

Fernanda Palhano Xavier de Fontes, Guillermo Andrade Barroso · Pierre Hellier INRIA Centre de Recherche Rennes Bretagne Atlantique

35042 Rennes Cedex

Tel.: +33.2.99.84.71.00

Fax: +33.2.99.84.71.71

E-mail: Pierre.Hellier@inria.fr 
offers a light, real-time, inexpensive, non-ionizing capability to image the surgical field and update the pre-operative planning. To fully benefit from this real-time capability, image processing methods also need to be real-time, which is also crucial for surgical applications. In this paper, a Bayesian version of the NL-means methods [3] is presented that allows to incorporate an ultrasound dedicated noise model. This denoising method was implemented using GPU technology and leads to a real-time denoising method. The paper is organized as follows: section 2 presents an overview of denoising methods, section 3 presents the modified NL-means method, section 4 describes the GPU implementation of the algorithm and section 5 presents results in terms of denoising quality and computation time.

\section{Related work}

In this paper, an efficient denoising method, dedicated to ultrasound images, is implemented using GPU capabilities. In this section, we briefly introduce related methods concerning image denoising, and GPU computation.

\subsection{Image denoising}

Many filters have been proposed for general image denoising. Ultrasound images are corrupted by speckle, a specific noise which is associated with coherent imaging systems. Many studies have been conducted to develop specific methods dedicated to ultrasound images. Denoising is a broad area and it would be out of the paper's scope to give an exhaustive survey. We refer the reader to $[21,9,6,5]$ for survey. Here, a short selection of denoising techniques is presented.

Adaptive filters Adaptive filters are based on the assumption that speckle is essentially a multiplicative noise. The most common adaptive filters are Lee's filter [19], Frost's filter [10], Kuan's filter [17]. The principle adopted by Lee and Kuan is very similar. Both seek to minimize the mean square error (MSE) between the true value of a pixel and its estimated value. The only difference between the two methods is that Lee utilizes a linear approximation for speckle while Kuan uses a nonlinear approximation. The Frost's filter is also a MSE filter, but assumes that the image is stationary. The median filter is a spatial filter that replaces the central pixel in a window with the median of all the pixel values in that window. Loupas [20] introduced a new approach to the adaptive median filter called adaptive weighted median filter (AWM). In this case, different weights are assigned for each pixel from the local content of the image. The filters mentioned above are easy to implement and present a good compromise between complexity and denoising quality.

Partial Differential Equations filters Some methods adapted the thermodynamic equation of heat to filter ultrasound images. Perona and Malik proposed an anisotropic filter with borders detection [23]. Alvarez et al.[1] also 
developed a filter based on the thermodynamic equation of heat which is called curvature motion. Total variation minimization scheme (TV) was introduced by Rudin, Osher and Fatemi [24]. The denoising problem is considered as a minimization problem with constraints, where the constraints are determined by statistical noise. In the original method, the removed noise is treated as an error. In practice, some structures and textures can be present in this error. Several groups have tried to avoid this effect $[25,22]$. Unlike the adaptive filters aforementioned, these methods are iterative and keep the location and accuracy of contours while smoothing the rest of the image.

\subsection{GPGPU}

Modern graphic cards have high computation and programmable capabilities for a reasonable monetary cost. The last generation of NVIDIA graphics card processors (GPU) have up to 240 processing units that can work in parallel with wide band memory. CUDA tools from NVIDIA allow to program graphics cards to solve intensive computation problems with a high-level langage. These facts push scientists to adopt the general purpose programing on graphic processor unit (GPGPU) in aim to attempt real-time level on intensive computation problems. This is the case for image and signal processing problems. [GPUCV] is a port of well-know OpenCV run-time libraries in GPU devices to accelerate filters execution and image processing basics tools.

Non-local means filters computation has good characteristics to be ported on GPU: every pixel computation needs a regular analyse of same type of zones over the image input. ImageDenoise SDK CUDA [15] implements a real-time generic non local means filter for $2 \mathrm{D}$ images which exploits CUDA $2 \mathrm{D}$ caches capabilities in textures memories into a GPU. [12] implements a non local means filter for deep data matrix of 3D points using CUDA. Recently, GPU has been used for medical image registration [18] and segmentation [4].

\section{Bayesian NL-means}

In this section, the original NL-means method [3] is briefly recalled, and the adaptation of the NL-means [7] to a dedicated ultrasound noise model [20].

\subsection{NL-means}

Here, the basic principles of the NL-means technique is presented. This method is based on a non-local paradigm, i.e., the restored intensity of a pixel is a weighted average of all pixels in the image (classically, a neighborhood around the considered pixel), weighted by the distance between the patches:

$$
N L(u)\left(x_{i}\right)=\sum_{x_{j} \in \Omega^{d i m}} w\left(x_{i}, x_{j}\right) u\left(x_{j}\right)
$$




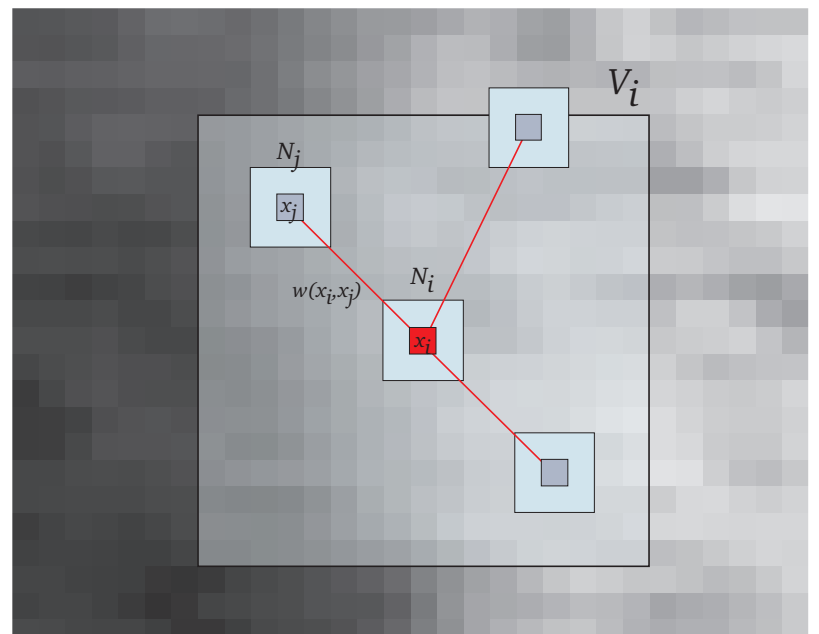

Fig. 1 Pixelwise $N L$-means filter $(d=1$ and $M=8)$. The restored value at pixel $x_{i}$ (in red) is the weighted average of all intensity values of pixels $x_{j}$ in the search volume $\Delta_{i}$. The weights are based on the similarity of the intensity neighborhoods (patches) $\mathbf{u}\left(\mathcal{N}_{i}\right)$ and $\mathbf{u}\left(\mathcal{N}_{j}\right)$.

where $w\left(x_{i}, x_{j}\right)$ is the weight assigned to value $u\left(x_{j}\right)$ for restoring the pixel $x_{i}$. More precisely, the weight evaluates the similarity between the intensities of the local neighborhoods (patches) $\mathcal{N}_{i}$ and $\mathcal{N}_{j}$ centered on pixels $x_{i}$ and $x_{j}$, such that $w\left(x_{i}, x_{j}\right) \in[0,1]$ and $\sum_{x_{j} \in \Omega^{\text {dim }}} w\left(x_{i}, x_{j}\right)=1$ (see Fig. 1 ). The size of the local neighborhood $\mathcal{N}_{i}$ and $\mathcal{N}_{j}$ is $(2 d+1)^{d i m}$. The traditional definition of the $N L$-means filter considers that the intensity of each pixel can be linked to pixel intensities of the whole image. For practical and computational reasons, the number of pixels taken into account in the weighted average is restricted to a neighborhood, that is a "search volume" $\Delta_{i}$ of size $(2 M+1)^{d i m}$, centered at the current pixel $x_{i}$.

For each pixel $x_{j}$ in $\Delta_{i}$, the Gaussian-weighted Euclidean distance $\|\cdot\|_{2, a}^{2}$ is computed between the two image patches $\mathbf{u}\left(\mathcal{N}_{j}\right)$ and $\mathbf{u}\left(\mathcal{N}_{i}\right)$ as explained in [3]. This distance is the traditional $L_{2}$-norm convolved with a Gaussian kernel of standard deviation $a$. The weights $w\left(x_{i}, x_{j}\right)$ are then computed as follows:

$$
w\left(x_{i}, x_{j}\right)=\frac{1}{Z_{i}} \exp -\frac{\left\|\mathbf{u}\left(\mathcal{N}_{i}\right)-\mathbf{u}\left(\mathcal{N}_{j}\right)\right\|_{2, a}^{2}}{h^{2}}
$$

where $Z_{i}$ is a normalization constant ensuring that $\sum_{x_{j} \in \Omega^{\text {dim }}} w\left(x_{i}, x_{j}\right)=1$ and $h$ acts as a filtering parameter controlling the decay of the exponential function.

\subsection{Bayesian NL-means}

The initial formulation of the NL-means filter relies on a $L_{2}$-norm between two patches, which relies on the assumption of an additive white Gaussian 
noise model. Unfortunately, the noise of ultrasound images cannot be considered as an additive white Gaussian noise. Here, a Bayesian formulation of the NL-means filter is used to incorporate an ultrasound dedicated noise model. We refer the reader to [7] for more details about this method.

The distribution of noise in ultrasound images has been largely studied in the literature so far and many models have been proposed, up to the utmost complexity. Among them, the Loupas noise model [20] has been successfully used in many studies. It reads as:

$$
u(x)=v(x)+v^{\gamma}(x) \eta(x)
$$

where $v(x)$ is the original image, $u(x)$ is the observed image, $\eta(x) \sim \mathcal{N}\left(0, \sigma^{2}\right)$ is a zero-mean Gaussian noise. This model is more flexible and less restrictive than the usual RF model and is able to capture reliably image statistics since the factor $\gamma$ depends on ultrasound devices and additional processing related to image formation. Contrary to additive white Gaussian noise model, the noise component in (3) is image-dependent. In [20], based on the experimental estimation of the mean versus the standard deviation in Log-compressed images, Loupas et al. have shown that $\gamma=0.5$ model fits better to data than the multiplicative model or the Rayleigh model. Since, this model has been used successfully in many studies $[16,2,29,11]$. Clearly, this model is relevant since it is confirmed that the speckle is higher in regions of high intensities versus regions of low intensities $[26,16]$.

In [14], a Bayesian formulation of the NL-means filter was proposed. Equivalent to the conditional mean estimator, it has been shown that an empirical estimator $\widehat{\mathbf{v}}\left(B_{i_{k}}\right)$ of a block $B_{i_{k}}$ can be defined as (see the appendix):

$$
\widehat{\mathbf{v}}\left(B_{i_{k}}\right)=\frac{\frac{1}{\left|\Delta_{i_{k}}\right|} \sum_{j=1}^{\left|\Delta_{i_{k}}\right|} \mathbf{v}\left(B_{j}\right) p\left(\mathbf{u}\left(B_{i_{k}}\right) \mid \mathbf{v}\left(B_{j}\right)\right)}{\frac{1}{\left|\Delta_{i_{k}}\right|} \sum_{j=1}^{\left|\Delta_{i_{k}}\right|} p\left(\mathbf{u}\left(B_{i_{k}}\right) \mid \mathbf{v}\left(B_{j}\right)\right)}
$$

where $p\left(\mathbf{u}\left(B_{i_{k}}\right) \mid \mathbf{v}\left(B_{j}\right)\right)$ denotes the probability density function (pdf) of $\mathbf{u}\left(B_{i_{k}}\right)$ given the noise free and unknown patches $\mathbf{v}\left(B_{j}\right)$.

Considering the Bayesian formulation and the Loupas noise model, a new formulation of the NL-means is proposed [7]. Assuming that

$$
u(x) \mid v(x) \backsim \mathcal{N}\left(v(x), v(x)^{2 \gamma} \sigma^{2}\right)
$$

leads to:

$$
p(u(x) \mid v(x)) \propto \exp -\frac{(u(x)-v(x))^{2}}{2 v(x)^{2 \gamma} \sigma^{2}} .
$$

Finally, this amount to substituting the traditional $L_{2}$-norm by the Pearson distance. We refer the reader to [7] for extensive details about this approach. 


\section{GPU Implementation}

Due to the high complexity of the NL-Means algorithm and the recent use of GPUs for massively parallel computation, we decided to use the GPU GeForce GTX 280 hardware version 1.3 to run our NL-Means filter adapted to ultrasound images. The large computational burden is mainly due to the computation of distances between each patch, which is used to later calculate the weight of a pixel. Since these distances are independent of each other, the algorithm is intrinsically parallel and particularly suited for such implementation. To develop the software, the NVIDIA CUDA was used. CUDA is a general purpose parallel computing architecture that uses a high level language compatible with $\mathrm{C} / \mathrm{C}++$.

Through CUDA, we can define functions, called kernels, that, when called, are executed $\mathrm{N}$ times in parallel by $\mathrm{N}$ different CUDA threads. These threads can be grouped into blocks. As the filtered images are $2 D$ images, we chose $2 D$ blocks for our implementation. The size of the blocks is an important parameter to computational time. Empirically, the fastest results were achieved partitioning each block into a $16 \times 16$ set of threads.

CUDA architecture has fast local memory called shared memory. This memory is faster than the GPU global memory, but is limited. For the GTX 280 there are 16384 bytes/block which are simultaneously used by shared memory and registers. Because of this limitation, we should select the most important data to copy into shared memory.

First, an image patch and its neighborhood are copied into shared memory. Second, the search area of each pixel are also copied into memory. This measure increases the performance, reducing runtime because of the reduction of global memory access. In ultrasound images, the area of interest containing image information is smaller than the size of the entire image. Thus, in order to further reduce the computational time, only points belonging to a given input mask were denoised, as illustrated in figure 2.

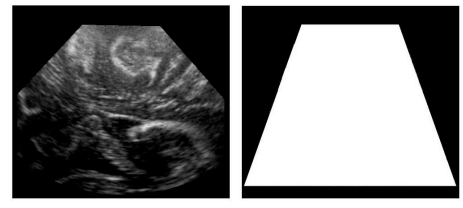

Fig. 2 Ultrasound image and corresponding mask. To reduce the computation time, only points belonging to the mask are denoised.

The program is composed of 8 kernels, 4 main kernels for NL-Means computation and another 4 auxiliary kernels to compute the mean and variance of the image. Also, a function implemented in $\mathrm{C}++$ is used to compute the smoothing parameter (h). Even though the program has 8 kernels, only 3 of them will be executed to filter an image. Two input parameters, determined by the user, specify how the kernels are called. There are two different implementations of the NL-means filter, a generic one [3] and a filter specifically 
adapted for ultrasound images [7]. Another input parameter which determines which kernels will be executed is the neighborhood size $N_{i}$. Because of limitations on shared memory size only two options were implemented: a neighborhood of $3 \times 3$ pixels or a neighborhood of $5 \times 5$ pixels.

The pseudocode in table 1 shows the main instructions that are executed when the programs runs. First of all, a preprocessing computation is performed. Two images are created: a map of local means and a map of local variances. These images will be used to select the relevant pixels on the input image. Once the preprocessing is done, the main kernel that implements the NL-means filter, will be executed. As mentioned above, an area of the image will be copied into shared memory. It is very important to synchronize after the copy to ensure that all threads are completed before proceeding to the next step which is computing the new value for each pixel using the NL-means algorithm.

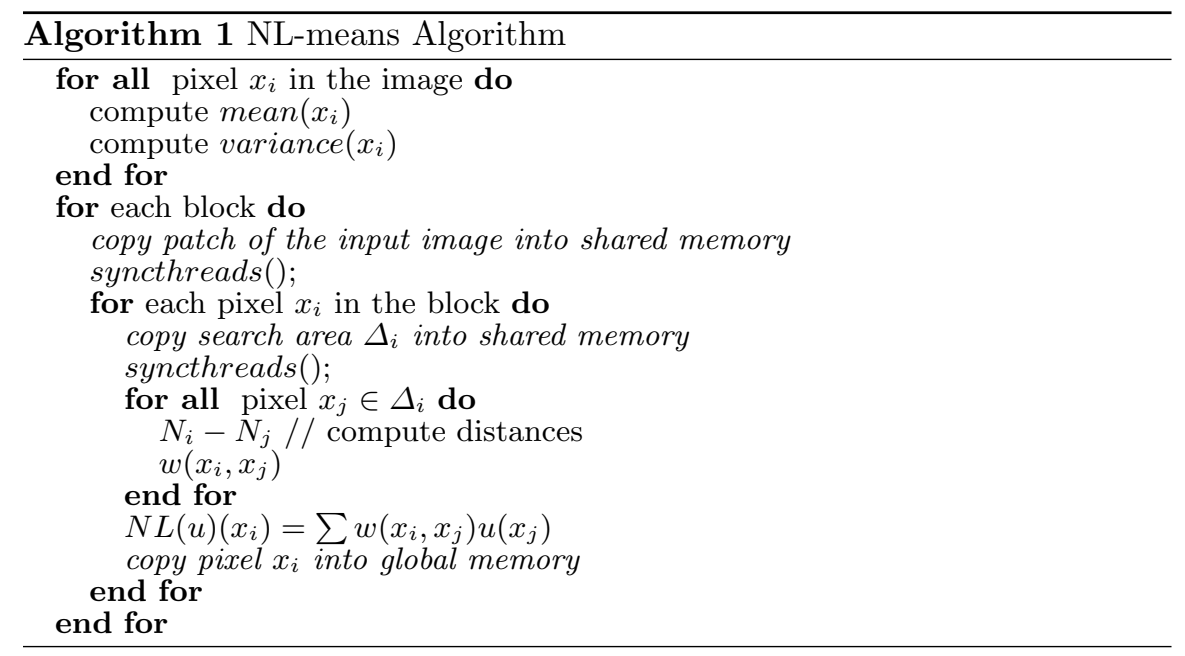

\section{Experiments}

In this section, results are presented, both in terms of denoising quality and computation time. Three methods are compared in what follows:

- A C ++ implementation of the NL-means method

- The CUDA SDK NL-means filter called imageDenoising [15]

- Our CUDA implementation of the Bayesian NL-means, called FUID (Fast Ultrasound Image Denoising). 
5.1 Denoising quality

\subsubsection{Qualitative evaluation}

The denoising methods were first tested on real intraoperative ultrasound images acquired during a neurosurgical procedure. The value of parameter $h$ was computed using an analysis of variance described in [8]. In this context, ultrasound images are acquired to be registered toward pre-operative MR images to update the surgical planning. Results are presented in figure 3 and show that the $\mathrm{C}++$ implementation and FUID lead to comparable results, exhibiting the desired properties of the NL-mens technique: homogeneous areas are smoothed and edges are preserved. The CUDA SDK implementation is visually less satisfactory. In a previously published paper [7], it has been shown objectively that the adapted NL-means performs better than the classical NL-means to denoise ultrasound images.

\subsubsection{Quantitative validation}

In order to evaluate the denoising filters with a relevant simulation of speckle noise, the validation framework proposed in [27], [28] was chosen for objective comparisons. This framework is based on Field II simulation [13]. Field II enables to generate realistic ultrasound images out of a echogeneicity map. As a result, the input geometry of the images is perfectly known and can be used for quantitative validation. The image test Cyst is composed of 3 constant classes $C_{r}$ presented in 4 . The result of the Field II simulation is converted to an 8 bit image of size $420 \times 315$ pixels. The geometry of the image is known but not the true value of the image without speckle. Therefore the authors introduced the ultrasound despeckling assessment index $(\tilde{Q})$ defined as:

$$
Q=\frac{\sum_{r \neq l}\left(\mu_{C_{r}}-\mu_{C_{l}}\right)^{2}}{\sum_{r} \sigma_{C_{r}}^{2}}
$$

Let us denote $\mu_{C_{r}}$ as the mean and $\sigma_{C_{r}}^{2}$ as the variance of class $C_{r}$ after denoising. To avoid the sensivity to image resolution, $Q$ is normalized by $Q_{i d}$. The new index $\tilde{Q}=Q / Q_{i d}$ is high if the applied filter is able to produce an image with well separated classes and small variances for each class. For these experiments we compared the FUID filter, the $\mathrm{C}++$ implementation of NL-means filter and the CUDA implementation of NL-means presented in the CUDA SDK (project named imageDenoising).

According to [27], [28], in this evaluation framework, the denoising quality is better when the $\tilde{Q}$ is high. FUID and $\mathrm{C}++$ implementation present similar $\tilde{Q}$. This result was expected and validates the FUID which is based on $\mathrm{C}++$ implementation.

In this experiment, the objects to be removed are composed of several pixels, thus the patch size is increased to evaluate the restoration performance of each object. The threshold $\left(\mu_{1}\right)$ was also modified in order to evaluate its influence. The parameters used for the test are given table 1 : 


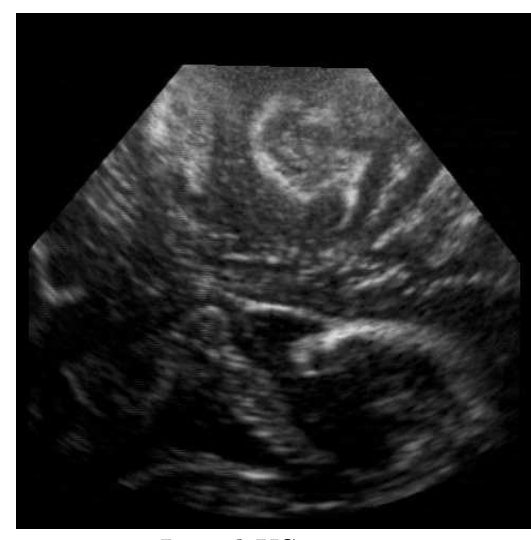

Initial US image

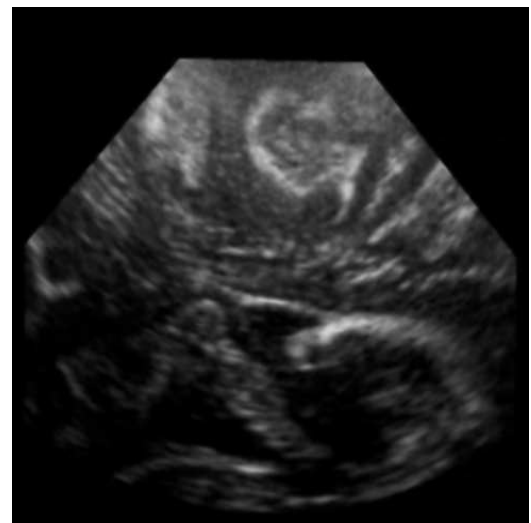

NL-means CUDA SDK

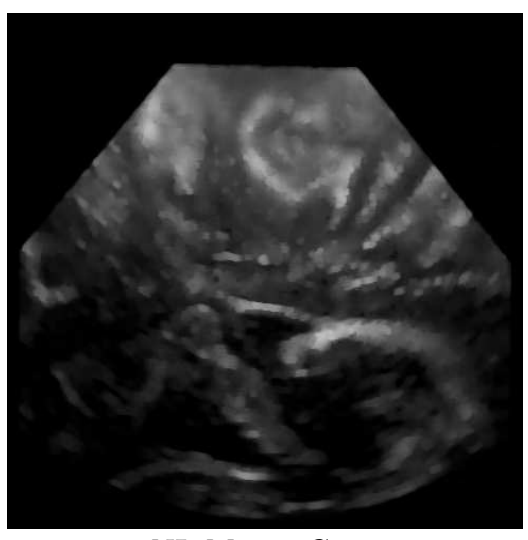

NL-Means $\mathrm{C}++$

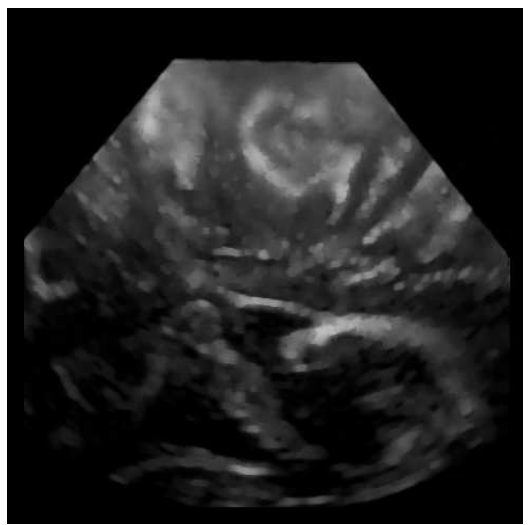

FUID

Fig. 3 Qualitative denoising results. A real intraoperative ultrasound was denoised with the three methods. Results show that the $\mathrm{C}++$ implementation and FUID lead to comparable results, exhibiting the desired properties of the NL-mens technique: homogeneous areas are smoothed and edges are preserved. The CUDA SDK implementation is visually less satisfactory.

\begin{tabular}{|c|c|c|c|c|}
\hline Filter & $\begin{array}{c}\text { Smoothing } \\
\text { parameter }\end{array}$ & $\begin{array}{c}\text { threshold } \\
\mu_{1}\end{array}$ & $\begin{array}{c}\text { Neighborhood } \\
\text { size }\end{array}$ & $\begin{array}{c}\text { Search } \\
\text { area }\end{array}$ \\
\hline FUID & 9.13 & 0.6 & $5 \times 5$ & $11 \times 11$ \\
\hline NL-means C++ & 9.13 & 0.6 & $5 \times 5$ & $11 \times 11$ \\
\hline NL-means CUDA & - & - & $5 \times 5$ & $11 \times 11$ \\
\hline
\end{tabular}

Table 1 Set of parameters used for computational time tests.

The denoised images and the quantitative results are given in Fig. 4. Compared to the NLMmeans CUDA SDK implementation, the proposed adaptations for ultrasound images improved the $\tilde{Q}$ of the denoised image. 


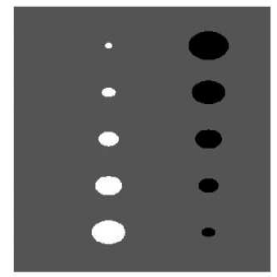

Cyst

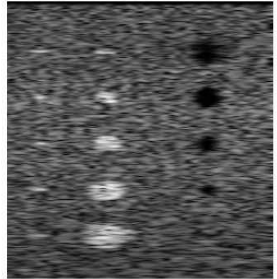

Field II simulation

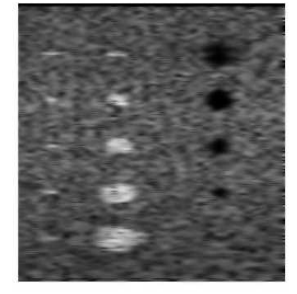

NL-means CUDA SDK $\tilde{Q}=1.38$

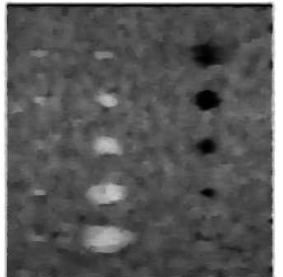

NL-Means $\mathrm{C}++$ $\tilde{Q}=2.10$

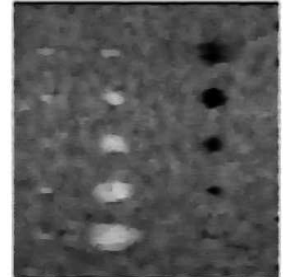

FUID

$\tilde{Q}=1.82$

Fig. 4 Results obtained with optimal set of parameters for $\tilde{Q}$.

\subsection{Computation time}

\subsubsection{Initial NL-means}

For the experiments, 5 ultrasound images were used. The size of the images ranged from $360 \times 288$ to $1080 \times 864$ pixels. We compared the computational time of FUID, C++ implementation of NL-means and CUDA SDK implementation of NL-means. Both FUID and imageDenoising were run using a 240 cores NVIDIA GTX 280 GPU. The $\mathrm{C}++$ implementation was performed on single core of a DualCore Intel Pentium CPU. The set of parameters of the 3 filters were the same. For a direct comparison with the NL-means implemented by imageDenoising, we used a classical version of FUID and C++ implementation (not adapted to ultrasound images).

The filters parameters included a neighborhood of $5 \times 5$ pixels and a search area of $11 \times 11$ pixels. The smoothing parameter was automatically computed for both the FUID and $\mathrm{C}++$ implementation. The results are given below (see figure 5 and table 3 ).

\subsubsection{Bayesian NL-means adapted to ultrasound images}

We also compared the FUID with $\mathrm{C}++$ implementation of NL-means both adapted for ultrasound images. Results are presented Fig. 6. We observe that FUID leads to the lowest computational time in both versions. Concerning the speedup of FUID, we observe in figure 7 a minimum speedup of 104.5 and a maximum speedup of 154.6 comparing with the $\mathrm{C}++$ implementation. 


\begin{tabular}{|c|c|c|c|c|}
\hline Filter & $\begin{array}{c}\text { Smoothing } \\
\text { parameter }\end{array}$ & $\begin{array}{c}\text { threshold } \\
\mu_{1}\end{array}$ & $\begin{array}{c}\text { Neighborhood } \\
\text { size }\end{array}$ & $\begin{array}{c}\text { Search } \\
\text { area }\end{array}$ \\
\hline FUID & 9.13 & 0.95 & $5 \times 5$ & $11 \times 11$ \\
\hline NL-means C++ & 9.13 & 0.95 & $5 \times 5$ & $11 \times 11$ \\
\hline NL-means CUDA & 9.13 & - & $5 \times 5$ & $11 \times 11$ \\
\hline
\end{tabular}

Table 2 Set of parameters used for computational time tests. The threshold does not apply to the NL-means CUDA implementation since the adaptive dictionary technique described in [8] was not used.

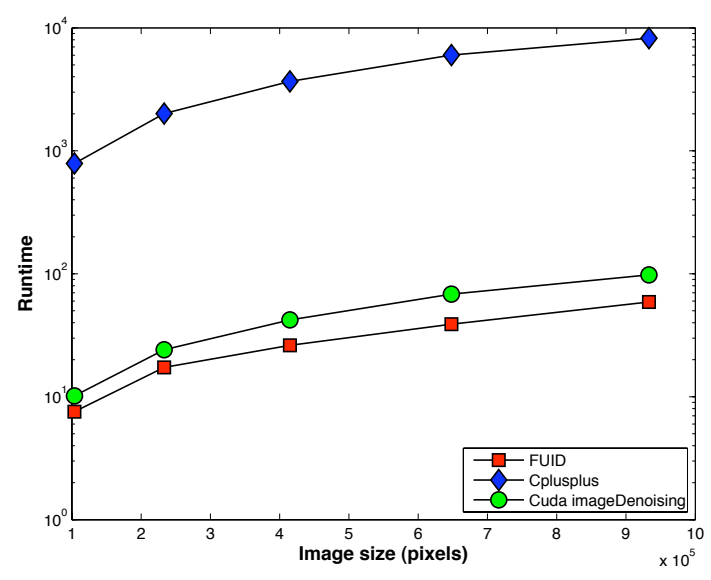

Fig. 5 Comparison: runtimes of FUID, C ++ implementation of NL-means and CUDA SDK implementation of NL-means. To draw the three methods on the same plot, a logarithmic scale was used for the computation time. Results indicate that the computation time increases in a similar way for all methods. Our implementation is faster in all cases.

Table 3 Computational time (ms) for different images size and improvement in percentage compared to the $\mathrm{C}++$ implementation. Results indicate that the speedup of CUDA implementations is approximately 100 compared to the $\mathrm{C}++$ implementation. In addition, our implementation is faster compared to the CUDA SDK one, with speedup factors ranging from $34 \%$ to $66 \%$ depending on the image size.

\begin{tabular}{|c|c|c|c|}
\hline & \multicolumn{3}{|c|}{ Computation time (ms) } \\
\hline $\begin{array}{l}\text { Image size } \\
\text { (pixels) }\end{array}$ & $\begin{array}{l}\text { NL-means's C++ } \\
\text { implementation }\end{array}$ & $\begin{array}{l}\text { NL-means's CUDA } \\
\text { SDK implementation }\end{array}$ & FUID \\
\hline $360 \times 288$ & 790 & $10.17(77 \%)$ & $\begin{array}{c}7.56 \\
(103 \%)\end{array}$ \\
\hline $540 \times 432$ & 2010 & $24.14(82 \%)$ & $\begin{array}{l}17.31 \\
(118 \%)\end{array}$ \\
\hline $720 \times 576$ & 3670 & $42.17(86 \%)$ & $\begin{array}{c}26.19 \\
(140 \%)\end{array}$ \\
\hline $900 \times 720$ & 6010 & $68.34(86 \%)$ & $\begin{array}{c}38.88 \\
(153 \%)\end{array}$ \\
\hline $1080 \times 864$ & 8240 & $98.06(83 \%)$ & $\begin{array}{c}58.94 \\
(141 \%)\end{array}$ \\
\hline
\end{tabular}




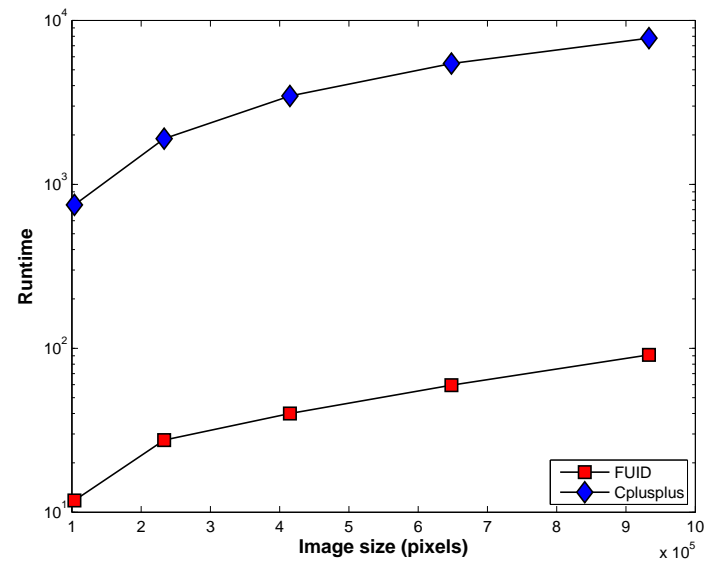

Fig. 6 Comparison: runtimes of FUID, C++ implementation of NL-means. The $\mathrm{y}$-axis is in logarithmic scale.

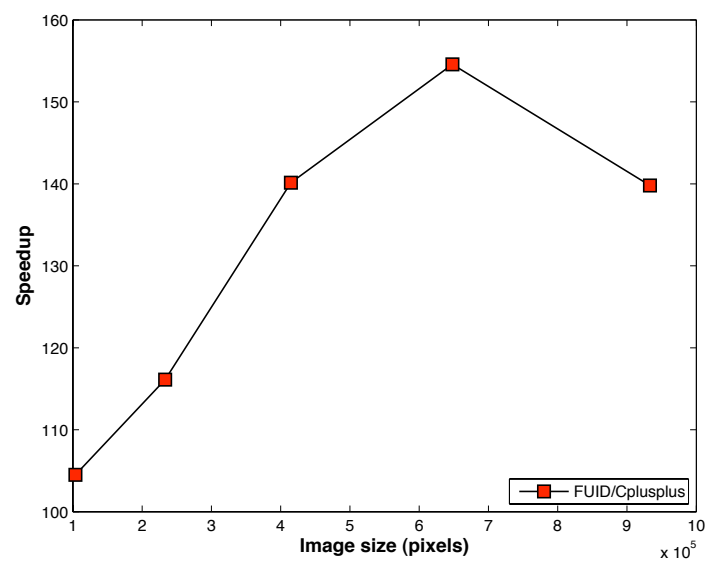

Fig. 7 Speedup of FUID/C++ implementation of NL-means

\section{Discussion and conclusion}

In this paper, a modified version of the NL-means algorithm, adapted to a dedicated ultrasound noise model, was presented. The proposed algorithm was implemented using GPU technology and was compared to the $\mathrm{C}++$ implementation, as well as to the NL-means approach implemented in the CUDA SDK. Results demonstrated that the proposed filter is very efficient in terms of denoising quality compared to state-of-the-art approaches.

In terms of computation time, the proposed method is real-time : for medium sized images, denoising was performed in less than $20 \mathrm{~ms}$, which is 
compatible with the vast majority of image processing workflows. In addition, our CUDA implementation was faster than the CUDA SDK implementation, with decrease varying between $34 \%$ to $66 \%$ depending on the image size.

Further work will investigate the use of the NL-means framework for additional image processing tasks. The patch-based framework is very general and can be declined for image super-resolution, image classification and registration. These NL-means based algorithms will also be particularly suited for GPU implementation.

Acknowledgements This work was partially funded by the ANR UScomp project.

\section{References}

1. L. Alvarez, P.L. Lions, and J.M. Morel. Image selective smoothing and edge detection by nonlinear diffusion. II, SIAM J. Numer. Anal., 29:845-866, 1992.

2. F. Argenti and G. Torricelli. Speckle suppression in ultrasonic images based on undecimated wavelets. EURASIP Journal on Advances in Signal Processing, 2003(5):470-478, 2003.

3. A. Buades, B. Coll, and JM. Morel. A review of image denoising algorithms, with a new one. Multiscale Modeling 83 Simulation, 4(2):490-530, 2005.

4. Joshua E. Cates, Aaron E. Lefohn, and Ross T. Whitaker. Gist: an interactive, gpu-based level set segmentation tool for 3d medical images. Medical Image Analysis, 8(3):217-231, 2004.

5. T.F. Chan, S. Osher, and J. Shen. The digital TV filter and nonlinear denoising. IEEE Transactions on Image Processing, 10(2):231-241, 2001.

6. Y. Chen, B.C. Vemuri, and L. Wang. Image denoising and segmentation via nonlinear diffusion. Computers and Mathematics with applications, 39(56):131-150, 2000 .

7. P. Coupé, P. Hellier, C. Kervrann, and C. Barillot. Nonlocal means-based speckle filtering for ultrasound images. IEEE Transactions on Image Processing, 18(10):2221-2229, 2009.

8. P. Coupé, P. Yger, S. Prima, P. Hellier, C. Kervrann, and C. Barillot. An Optimized Blockwise Non Local Means Denoising Filter for 3D Magnetic Resonance Images. IEEE Transactions on Medical Imaging, 27(4):425-441, 2008.

9. M. Elad and M. Aharon. Image denoising via sparse and redundant representations over learned dictionaries. IEEE Transactions on Image Processing, 15(12):3736-3745, 2006.

10. V.S. Frost, J.A Stiles, K.S Shanmugan, and J.C. Holtzman. A model for radar images and its application to adaptive digital filtering of multiplicative noise. IEEE Transactions on Pattern Analysis and Machine Intelligence, 2:157-65, 1982.

11. X. Hao, S. Gao, and X. Gao. A novel multiscale nonlinear thresholding method for ultrasonic speckle suppressing. IEEE Transactions on Medical Imaging, 18(9):787-794, 1999.

12. B. Huhle, T. Schairer, P. Jenke, and W. Straßer. Robust non-local denoising of colored depth data. In IEEE Computer Society Conference on Computer Vision and Pattern Recognition Workshops, 2008. CVPR Workshops 2008, pages 1-7, 2008.

13. J. A. Jensen. Field: A program for simulating ultrasound systems. Medical $\mathcal{E}$ Biological Engineering 83 Computing, 34:351-353, 1996.

14. C. Kervrann, J. Boulanger, and P. Coupï $; \frac{1}{2}$. Bayesian non-local means filter, image redundancy and adaptive dictionaries for noise removal. In Proc. Conf. Scale-Space and Variational Meth. (SSVM'07), pages 520-532, Ischia, Italy, June 2007.

15. A. Kharlamov and V. Podlozhnyuk. Image Denoising. 
16. K. Krissian, K. Vosburgh, R. Kikinis, and C-F. Westin. Speckle-constrained anisotropic diffusion for ultrasound images. In IEEE Computer Society Conference on Computer Vision and Pattern Recognition, June 2005.

17. D.T Kuan, A.A. Sawchuck, T.C Strand, and P.Chavel. Adaptive noise smoothing filter for images with signal-dependent noise. IEEE Transactions on Pattern Analysis and Machine Intelligence, 7(2):165-177, 1985.

18. A. Kubias, F. Deinzer, T. Feldmann, D. Paulus, B. Schreiber, and Th. Brunner. 2d/3d image registration on the gpu. Pattern Recognition and Image Analysis, 18(3):381-389, 2008.

19. J. S. Lee. Digital image enhancement and noise filtering by use of local statistics. IEEE Transactions on Pattern Analysis and Machine Intelligence, 2:165$168,1980$.

20. T. Loupas, W.N. McDicken, and P.L. Allan. An adaptive weighted median filter for speckle suppression in medical ultrasound image. IEEE T. Circ. Syst., 36:129-135, 1989.

21. M.C. Motwani, M.C. Gadiya, R.C. Motwani, and F.C. Harris Jr. Survey of image denoising techniques. In Proceedings of GSPx, pages 27-30. Citeseer, 2004.

22. S. Osher, M. Burger, D. Goldfarb, J. Xu, and W. Yin. An iterative regularization method for total variation-based image restoration. Multiscale Model. Simul., 4(2):460-489, 2005.

23. P. Perona and J. Malik. Scale-space and edge detection using anisotropic diffusion. IEEE Transactions on Pattern Analysis and Machine Intelligence, 12(7):629-639, 1990.

24. L.I. Rudin, S. Osher, and E. Fatemi. Nonlinear total variation based noise removal algorithms. Physica D, 60:259-268, 1992.

25. E. Tadmor, S. Nezzar, and L. Vese. A multiscale image representation using hierarchical (BV, $\left.L^{2}\right)$ decompositions. Multiscale Model. Simul., 2(4):554-579, 2004.

26. Z. Tao, H. D. Tagare, and J. D. Beaty. Evaluation of four probability distribution models for speckle in clinical cardiac ultrasound images. IEEE Transactions on Medical Imaging, 25(11):1483-1491, 2006.

27. P. C. Tay, S. T. Acton, and J. A. Hossack. A stochastic approach to ultrasound despeckling. In Biomedical Imaging: Nano to Macro, 2006. 3rd IEEE International Symposium on, pages 221-224, 2006.

28. P. C. Tay, S. T. Acton, and J. A. Hossack. Ultrasound despeckling using an adaptive window stochastic approach. In IEEE International Conference on Image Processing, pages 2549-2552, 2006.

29. M. P. Wachowiak, A. S. Elmaghraby, R. Smolíkova, and J. M. Zurada. Classification and estimation of ultrasound speckle noise with neural networks. In IEEE International Symposium on Bio-Informatics and Biomedical Engineering (BIBE'00), pages 245-252, 2000. 